\title{
Multiple Faults Detection and Isolation via Decentralized Sliding Mode Observer for Reconfigurable Manipulator
}

\author{
Bo Zhao*, Chenghao $\mathrm{Li}^{* *}$, Tianhao $\mathrm{Ma}^{* * *}$ and Yuanchun $\mathrm{Li}^{\dagger}$
}

\begin{abstract}
This paper considers a decentralized multiple faults detection and isolation (FDI) scheme for reconfigurable manipulators. Inspired by their modularization property, a global sliding mode (GSM) based stable adaptive fuzzy decentralized controller is investigated for the system in fault free, while for the system suffering from multiple faults (actuator fault and sensor fault), the decentralized sliding mode observer (DSMO) is employed to detect their occurrence. Hereafter, the time and location of faults can be determined by a fault isolation scheme via a bank of DSMOs. Finally, the effectiveness of the proposed schemes in controlling, detecting and isolating faults is illustrated by the simulations of two 3-DOF reconfigurable manipulators with different configurations successfully.
\end{abstract}

Keywords: Reconfigurable manipulator, Multiple faults detection and isolation, Decentralized sliding mode observer, Decentralized control

\section{Introduction}

Reconfigurable manipulators [1], which are composed of a set of modules in standard size and interface, can be assembled into different configurations and geometries to undertake a variety of unknown or changeable tasks, such as the space exploration, smart manufacturing, high risk operations, and battle fields. However, working for a long time in the environments human can not be involved directly, faults will inevitably occur in actuator, sensor or other components, which may lead the control system to unstable or even destroyed. Therefore, the safety and reliability of reconfigurable manipulators becomes an urgent demand, and the efforts on its FDI are essential.

As a nonlinear and strong interconnected system, the controller design for reconfigurable manipulator is a quite active research field, and different approaches have been proposed during the past decades. The main challenging work lies in the dynamic parameter uncertainties caused by reconfiguration, friction, varying payloads and interconnection among joints. To against them, several schemes were developed for reconfigurable manipulators [2-4], but they need to be redesigned when adds, removes or changes modules [5]. In contrast, the modular distributed control technology in [6] was more feasible. However, the communication time delay among the modules will lead to

$\dagger$ Corresponding Author: Department of Control Science and Engineering, Changchun University of Technology, China. (liyc@ccut.edu.cn)

* The State Key Laboratory of Management and Control for Complex Systems, Institute of Automation, Chinese Academy of Sciences, Beijing 100190, China.

** Product Development, FAW Car Co., Ltd, Changchun 130012 China.

*** Department of Control Science and Engineering, Changchun University of Technology, Changchun 130012, China.

Received: November 13, 2014; Accepted: July 8, 2015 the inaccurate performance [7]. Take the modularization property and interconnection into account, decentralized control strategy was established [8-12] to overcome these shortcomings. It can not only effectively reduce the computational burden in centralized control, but also avoid communication time delay in distributed control, thus it is more feasible to implement to reconfigurable manipulators.

On the other hand, the investigated FDI approaches [13] can be essentially grouped into three main categories. Model-based FDI is a powerful solution [14]. Based on the estimation error obtained from the strain gauge sensor signal and the reaction force observer [15], the sensor fault can be detected, and then the faulty sensor signal was exchanged by the estimated one to maintain the stable control performance. In the presence of parametric uncertainties and actuator faults, a robust reliable control [16] for a near space vehicle was studied based on the fuzzy state-space observer. Based on signal processing technologies, a three latent space transformation based principal component analysis [17] has been effectively applied to detect the fault in the industrial processes with a large number of high correlated variables. In [18], an artificial immunization algorithm was used to optimize the parameters in support vectors machine, it can avoid the premature convergence and guarantee the variety of solutions. Knowledge-based FDI is another effective method. A back propagation neural network $(\mathrm{NN})$ was presented [19] to synthesize fault detection components based on the data collected in the training of autonomous robots. By virtue of soft computing techniques, an integrator of NN and fuzzy logic was proposed [20] for FDI of robot manipulators.

For reconfigurable manipulator systems, only several FDI schemes have been carried out. Inspired by the relationship between power efficiency and operation health 
conditions, a power efficiency estimation based health monitoring and fault detection technique was developed for modular and reconfigurable robots (MRR) with a joint torque sensor [21]. By the filtered torque estimation, [22] proposed a distributed fault detection scheme with torque sensing for MRR. In [23], a hybrid controller has been constructed by a fault estimator and an impulse controller. Zhao et al. [24] compared the user defined threshold to the residual between the actual velocity and the observed one, to detect the fault occurrence or not, and the unknown input state observer was exploited for fault identification.

Sliding mode observer (SMO) [25-28] is a good idea for FDI. In [29], SMO was employed to overcome the limitation that linear observer schemes cannot be utilized for sensor fault reconstruction. The authors in [30] performed a modular fault detection scheme, which did not require motion states of any other modules. In [31], the sensor fault was converted into pseudo-actuator fault scenario by a filter, and a decentralized sliding mode observer was recruited for the position sensor or velocity sensor fault identification.

This presented idea develops an adaptive fuzzy decentralized controller based on GSM for reconfigurable manipulators. For the purpose of FDI, the DSMO is employed to detect and isolate multiple faults, which can be judged when the observation errors beyond the predefined threshold. Hereafter, the time and location of fault occurrence can be decided by the fault isolation scheme. The main contributions of this work are as follows. (i) According to their modularization property, the decentralized controller is more feasible to reconfigurable manipulators, it implies that it is no need to redesign the controller when adds or removes the modules. (ii) The interconnection term is compensated to improve the joint tracking accuracy. (iii) The actuator and sensor fault can be detected precisely even they occur simultaneously. (iv) The fault time and location can be decided by isolating the multiple faults successfully.

The remainder of this paper is organized as follows. In Section 2, the dynamic models of reconfigurable manipulators with/without faults and the control objective are described. And then the decentralized controller is designed in Section 3. Subsequently, the FDI scheme is given in detail in Section 4. Hereafter, the effectiveness of the proposed methods is confirmed by the simulations in Section 5. Finally, the conclusions are drawn in Section 6.

\section{Problem Statement}

The dynamic model of $n$-DOF reconfigurable manipulator with joint dynamic friction obtained by Newton-Euler formulation should be described as

$$
M(q) \ddot{q}+C(q, \dot{q}) \dot{q}+G(q)+F(q, \dot{q})=u
$$

where $q \in R^{n}$ is the vector of joint displacements, $M(q) \in R^{n \times n}$ is the positive definite inertia matrix, $C(q, \dot{q}) \dot{q} \in R^{n}$ is the Coriolis and centripetal force, $G(q) \in R^{n}$ is the gravity term, $F(q, \dot{q}) \in R^{n}$ is the vector of joint dynamic friction torques, and $u \in R^{n}$ is the applied joint torque.

For the development of decentralized control, each joint is considered as a subsystem of the entire manipulator system interconnected by coupling torques. By separating terms only depending on local variables $\left(q_{i}, \dot{q}_{i}, \ddot{q}_{i}\right)$ from those terms of other joint variables, each subsystem dynamical model can be formulated in joint space as

$$
\begin{aligned}
& M_{i}\left(q_{i}\right) \ddot{q}_{i}+C_{i}\left(q_{i}, \dot{q}_{i}\right) \dot{q}_{i}+G_{i}\left(q_{i}\right)+ \\
& F_{i}\left(q_{i}, \dot{q}_{i}\right)+Z_{i}(q, \dot{q}, \ddot{q})=u_{i}
\end{aligned}
$$

where $q_{i}, \dot{q}_{i}, \ddot{q}_{i}, G_{i}(q), F_{i}\left(q_{i}, \dot{q}_{i}\right)$ and $u_{i}$ are the $i$-th element of the vectors $q, \dot{q}, \ddot{q}, G(q), F(q, \dot{q})$ and $u$, respectively. $M_{i j}(q)$ and $C_{i j}(q, \dot{q})$ are the $i j$-th element of the matrices $M(q)$ and $C(q, \dot{q})$, respectively.

Property 1: For scalar functions $M_{i}\left(q_{i}\right)$ and $C_{i}\left(q_{i}, \dot{q}_{i}\right)$, the following condition holds [9]

$$
s_{i}^{T}\left(\dot{M}_{i}-2 C_{i}\right) s_{i}=0
$$

Let $x_{i}=\left[\begin{array}{ll}x_{i 1} & x_{i 2}\end{array}\right]^{T}=\left[\begin{array}{ll}q_{i} & \dot{q}_{i}\end{array}\right]^{T}(i=1,2, \cdots, n)$, (2) can be expressed as the following state space equation

$$
S_{i}:\left\{\begin{array}{l}
\dot{x}_{i}=A_{i} x_{i}+B_{i}\left[f_{i}\left(q_{i}, \dot{q}_{i}\right)+g_{i}\left(q_{i}\right) u_{i}+h_{i}(q, \dot{q}, \ddot{q})\right] \\
y_{i}=C_{i} x_{i}
\end{array}\right.
$$

where $x_{i}$ and $y_{i}$ are the state vector and output vector of subsystem $S_{i}$, respectively. And

$$
\begin{aligned}
& A_{i}=\left[\begin{array}{ll}
0 & 1 \\
0 & 0
\end{array}\right] \quad B_{i}=\left[\begin{array}{l}
0 \\
1
\end{array}\right] \quad C_{i}=\left[\begin{array}{ll}
1 & 0
\end{array}\right] \\
& f_{i}\left(q_{i}, \dot{q}_{i}\right)=M_{i}^{-1}\left(q_{i}\right)\left[-C_{i}\left(q_{i}, \dot{q}_{i}\right) \dot{q}_{i}-G_{i}\left(q_{i}\right)-F_{i}\left(q_{i}, \dot{q}_{i}\right)\right] \\
& g_{i}\left(q_{i}\right)=M_{i}^{-1}\left(q_{i}\right) \\
& h_{i}(q, \dot{q}, \ddot{q})=-M_{i}^{-1}\left(q_{i}\right) Z_{i}(q, \dot{q}, \ddot{q})
\end{aligned}
$$

The dynamic model of the $i$-th subsystem exhibits both actuator and sensor faults should be described by

$$
S_{i f}:\left\{\begin{aligned}
\dot{x}_{i}= & A_{i} x_{i}+B_{i}\left[f_{i}\left(q_{i}, \dot{q}_{i}\right)+g_{i}\left(q_{i}\right) u_{i}+\right. \\
& \left.h_{i}(q, \dot{q}, \ddot{q})+f_{i a}\left(q_{i}, \dot{q}_{i}, u_{i}\right)\right] \\
y_{i}= & C_{i} x_{i}+f_{i s}\left(q_{i}, \dot{q}_{i}\right)
\end{aligned}\right.
$$




$$
\begin{gathered}
f_{i a}\left(q_{i}, \dot{q}_{i}, u_{i}\right)=\alpha\left(t-T_{i a}\right) M_{i}^{-1}\left(q_{i}\right) \psi_{i}\left(q_{i}, \dot{q}_{i}, u_{i}\right) \\
f_{i s}\left(q_{i}, \dot{q}_{i}\right)=\alpha\left(t-T_{i s}\right) \phi_{i}\left(q_{i}, \dot{q}_{i}\right)
\end{gathered}
$$

where $\alpha\left(t-T_{i a}\right)$ and $\alpha\left(t-T_{i s}\right)$ are step functions, $T_{i a}$ and $T_{i s}$ are the occurrence times of actuator and sensor fault of the $i$-th subsystem, respectively. $\psi_{i}\left(q_{i}, \dot{q}_{i}, u_{i}\right)$ and $\phi_{i}\left(q_{i}, \dot{q}_{i}\right)$ are the actuator and sensor fault functions, respectively, $f_{i a}\left(q_{i}, \dot{q}_{i}, u_{i}\right)$ and $f_{i s}\left(q_{i}, \dot{q}_{i}\right)$ are the actuator and sensor fault terms, respectively.

Assumption 1: The actuator and sensor fault terms $f_{i a}\left(q_{i}, \dot{q}_{i}, u_{i}\right)$ and $f_{i s}\left(q_{i}, \dot{q}_{i}\right)$ are unknown but norm bounded with $\left\|f_{i a}\left(q_{i}, \dot{q}_{i}, u_{i}\right)\right\| \leq \delta_{i}$ and $\left\|f_{i s}\left(q_{i}, \dot{q}_{i}\right)\right\| \leq \rho_{i}$, where $\delta_{i}$ and $\rho_{i}$ are positive constants.

The objective of this work is to detect and isolate the multiple faults occur in the $i$-th subsystem in real-time based on the local joint information. In other words, fault detection is to determine the fault occurrence time, and fault isolation decides the fault location.

\section{Decentralized Controller Design}

The traditional sliding mode surface exists approaching mode and sliding mode during the whole sliding process, while the robustness of parameter uncertainties and external disturbances only present during the sliding mode. Yet the global sliding surface [32-33] can drive the system state to the sliding surface at the very beginning, the reaching interval can be eliminated.

In this section, an adaptive fuzzy controller only depends on the local joint information is designed to handle the joint tracking control of reconfigurable manipulators in fault free.

Define the tracking error $e_{i}$ as

$$
e_{i}=q_{i d}-q_{i}
$$

Then define the global dynamic sliding mode surface as

$$
s_{i}=\dot{e}_{i}+\lambda_{i} e_{i}-f_{i}(t)
$$

where $\lambda_{i}>0, f_{i}(t)$ is the designed function for reaching the global sliding mode, which satisfies the following three conditions:

(i) the initial $f_{i}(0)=\dot{e}_{i 0}+c_{i} e_{i 0}$, where $e_{i 0}$ and $\dot{e}_{i 0}$ are the initial values of $e_{i}$ and $\dot{e}_{i}$, respectively.

(ii) $\lim _{t \rightarrow \infty} f_{i}(t)=0$.

(iii) $\dot{f}_{i}(t)$ is existence.

According to the above conditions, $f_{i}(t)$ should be

$$
f_{i}(t)=f_{i}(0) e^{-k_{i} t}\left(k_{i}>0\right)
$$

Yields,

$$
\dot{q}_{i}=-s_{i}+\dot{q}_{i d}+\lambda_{i} e_{i}-f_{i}(t)
$$

Assumption 2: The desired trajectories $q_{i d}$ are twice differentiable and bounded as

$$
\left\|\begin{array}{l}
q_{i d} \\
\dot{q}_{i d} \\
\ddot{q}_{i d}
\end{array}\right\| \leq q_{i A}
$$

where $q_{i A}$ is a known positive scalar.

Assumption 3: The interconnection term $Z_{i}(q, \dot{q}, \ddot{q})$ is explicitly bounded by [24]

$$
Z_{i}(q, \dot{q}, \ddot{q}) \leq \sum_{j=1}^{n} d_{i j} S_{j}
$$

where $d_{i j} \geq 0, S_{j}=1+\left|s_{j}\right|+\left|s_{j}\right|^{2}$.

Define $x_{i}=\left(q_{i}, \dot{q}_{i}, q_{i d}, \dot{q}_{i d}, \ddot{q}_{i d}\right)^{T}$, then substituting the time derivative of (13) into (2), one can obtain that

$$
\begin{aligned}
M_{i}\left(q_{i}\right) \dot{s}_{i}= & M_{i}\left(q_{i}\right)\left(\ddot{q}_{i d}-\ddot{q}_{i}+\lambda_{i} \dot{e}_{i}-\dot{f}_{i}(t)\right) \\
= & -C_{i}\left(q_{i}, \dot{q}_{i}\right) s_{i}-u_{i}+Z_{i}(q, \dot{q}, \ddot{q})+\phi_{i}\left(x_{i}\right) \\
& -C_{i}\left(q_{i}, \dot{q}_{i}\right) f_{i}(t)-M_{i}\left(q_{i}\right) \dot{f}_{i}(t)
\end{aligned}
$$

where $\phi_{i}\left(x_{i}\right)$ is the defined nonlinear function defined as $\phi_{i}\left(x_{i}\right)=M_{i}\left(q_{i}\right)\left(\ddot{q}_{i d}+\lambda_{i} \dot{e}_{i}\right)+C_{i}\left(q_{i}, \dot{q}_{i}\right)\left(\dot{q}_{i d}+\lambda_{i} e_{i}\right)+G_{i}\left(q_{i}\right)$.

Now, the fuzzy logic systems are adopted to approximate the bounded nonlinear functions $\phi_{i}\left(x_{i}\right)$, $M_{i}\left(q_{i}\right)$ and $C_{i}\left(q_{i}, \dot{q}_{i}\right)$, yields

$$
\begin{gathered}
\phi_{i}\left(x_{i}\right)=\theta_{i \phi}^{T} \sigma_{i \phi}\left(x_{i}\right)+\varepsilon_{i 1} \\
M_{i}\left(q_{i}\right)=\theta_{i M}^{T} \sigma_{i M}\left(q_{i}\right)+\varepsilon_{i 2} \\
C_{i}\left(q_{i}, \dot{q}_{i}\right)=\theta_{i C}^{T} \sigma_{i C}\left(q_{i}, \dot{q}_{i}\right)+\varepsilon_{i 3}
\end{gathered}
$$

where $\varepsilon_{i 1}, \varepsilon_{i 2}$ and $\varepsilon_{i 3}$ are the fuzzy logic system approximate errors, and $\theta_{i \phi}, \theta_{i M}, \theta_{i C}$ is the optimal parameter vectors satisfy the following conditions, respectively.

$$
\begin{aligned}
& \theta_{i \phi}=\underset{\hat{\theta}_{i \phi} \in \Omega_{i \phi}}{\arg \min }\left\{\operatorname{Sup}\left|\hat{\phi}_{i}\left(x_{i}, \hat{\theta}_{i \phi}\right)-\phi_{i}\left(x_{i}\right)\right|\right\} \\
& \theta_{i M}=\underset{\hat{\theta}_{i M} \in \Omega_{i M}}{\arg \min }\left\{\operatorname{Sup}\left|\hat{M}_{i}\left(q_{i}, \hat{\theta}_{i M}\right)-M_{i}\left(q_{i}\right)\right|\right\} \\
& \theta_{i C}=\underset{\hat{\theta}_{i C} \in \Omega_{i C}}{\arg \min }\left\{\begin{array}{c}
\left.\operatorname{Sup}\left|\hat{C}_{i}\left(q_{i}, \dot{q}_{i}, \hat{\theta}_{i C}\right)-C_{i}\left(q_{i}, \dot{q}_{i}\right)\right|\right\} \\
\left(q_{i}, \dot{q}_{i}\right)^{T} \in U_{i C}
\end{array}\right\}
\end{aligned}
$$

where $\Omega_{i \phi}, \Omega_{i M}, \Omega_{i C}, U_{i \phi}, U_{i M}$ and $U_{i C}$ are the constraint 
set of $\hat{\theta}_{i \phi}, \hat{\theta}_{i M}, \hat{\theta}_{i C}, x_{i}, q_{i}$ and $\left(q_{i}, \dot{q}_{i}\right)^{T}$, respectively. $\hat{\phi}_{i}\left(x_{i}, \hat{\theta}_{i \phi}\right), \hat{M}_{i}\left(q_{i}, \hat{\theta}_{i M}\right)$ and $\hat{C}_{i}\left(q_{i}, \dot{q}_{i}, \hat{\theta}_{i C}\right)$ are the estimations of $\phi_{i}\left(x_{i}\right), M_{i}\left(q_{i}\right)$ and $C_{i}\left(q_{i}, \dot{q}_{i}\right)$ that could be expressed as

$$
\begin{gathered}
\hat{\phi}_{i}\left(x_{i}\right)=\hat{\theta}_{i \phi}^{T} \sigma_{i \phi}\left(x_{i}\right) \\
\hat{M}_{i}\left(q_{i}\right)=\hat{\theta}_{i M}^{T} \sigma_{i M}\left(q_{i}\right) \\
\hat{C}_{i}\left(q_{i}, \dot{q}_{i}\right)=\hat{\theta}_{i C}^{T} \sigma_{i C}\left(q_{i}, \dot{q}_{i}\right)
\end{gathered}
$$

where $\hat{\theta}_{i \phi}, \hat{\theta}_{i M}$ and $\hat{\theta}_{i C}$ are adjustable parameters to estimate $\theta_{i \phi}, \theta_{i M}$ and $\theta_{i C}$, respectively.

Define the minimum approximate error as

$$
\varepsilon_{i}=\varepsilon_{i 1}\left(x_{i}\right)-\varepsilon_{i 2}\left(q_{i}\right) \dot{f}_{i}(t)-\varepsilon_{i 3}\left(q_{i}, \dot{q}_{i}\right) f_{i}(t)
$$

Assumption 4: The approximate error of fuzzy logic system satisfies $\left|\varepsilon_{i}\right| \leq \varepsilon_{0}$, where $\varepsilon_{0}>0$.

The GSM based adaptive fuzzy decentralized control law is designed as

$$
\begin{gathered}
u_{i}=u_{i 0}+u_{i c} \\
u_{i 0}=\hat{\theta}_{i \phi}^{T} \sigma_{i \phi}\left(x_{i}\right)-\hat{\theta}_{i C}^{T} \sigma_{i C}\left(q_{i}, \dot{q}_{i}\right) f_{i}(t) \\
-\hat{\theta}_{i M}^{T} \sigma_{i M}\left(q_{i}\right) \dot{f}_{i}(t)+k_{i} s_{i} \\
u_{i c}=\varepsilon_{0} \operatorname{sgn}\left(s_{i}\right)+\hat{\delta}_{i} \operatorname{sgn}\left(s_{i}\right) S_{i}
\end{gathered}
$$

where $k_{i}$ is positive constant, $\hat{\delta}_{i}$ is the estimated value of $\delta_{i}=n \max _{i j}\left\{d_{i j}\right\}$, then the estimation errors are defined as

$$
\begin{gathered}
\tilde{\theta}_{i \phi}=\theta_{i \phi}-\hat{\theta}_{i \phi} \\
\tilde{\theta}_{i M}=\theta_{i M}-\hat{\theta}_{i M} \\
\tilde{\theta}_{i C}=\theta_{i C}-\hat{\theta}_{i C} \\
\tilde{\delta}_{i}=\delta_{i}-\hat{\delta}_{i}
\end{gathered}
$$

where $\hat{\theta}_{i \phi}, \hat{\theta}_{i M}, \hat{\theta}_{i C}$ and $\hat{\delta}_{i}$ are updated by

$$
\begin{gathered}
\dot{\hat{\theta}}_{i \phi}=\eta_{i \phi} s_{i} \sigma_{i \phi}\left(x_{i}\right) \\
\dot{\hat{\theta}}_{i M}=\eta_{i M} s_{i} \dot{f}_{i}(t) \sigma_{i M}\left(q_{i}\right) \\
\dot{\hat{\theta}}_{i C}=\eta_{i C} s_{i} f_{i}(t) \sigma_{i C}\left(q_{i}, \dot{q}_{i}\right) \\
\dot{\hat{\delta}}_{i}=\eta_{i \delta}\left|s_{i}\right| S_{i}
\end{gathered}
$$

Theorem 1: Consider the subsystem dynamic model (2) with assumptions 2-4, utilize the control law (27)-(29) and adaptive laws (34)-(37), all the variables of the closed-loop system are guaranteed to be bounded, and the $H_{\infty}$ tracking performance is satisfied.
Proof: Choose the Lyapunov function candidate, i.e.,

$$
\begin{aligned}
V=\sum_{i=1}^{n}[ & \frac{1}{2} M_{i}\left(q_{i}\right) s_{i}^{2}+\frac{1}{2 \eta_{i \phi}} \tilde{\theta}_{i \phi}^{T} \tilde{\theta}_{i \phi}+\frac{1}{2 \eta_{i M}} \tilde{\theta}_{i M}^{T} \tilde{\theta}_{i M} \\
& \left.+\frac{1}{2 \eta_{i C}} \tilde{\theta}_{i C}^{T} \tilde{\theta}_{i C}+\frac{1}{2 \eta_{i \delta}} \tilde{\delta}_{i}^{2}\right]
\end{aligned}
$$
is

Take property 1 into account, the time derivative of (38)

$$
\begin{aligned}
\dot{V}= & \sum_{i=1}^{n}\left[s _ { i } \left(-C_{i}\left(q_{i}, \dot{q}_{i}\right) s_{i}-u_{i}+Z_{i}(q, \dot{q}, \ddot{q})+\phi_{i}\left(x_{i}\right)\right.\right. \\
& \left.-C_{i}\left(q_{i}, \dot{q}_{i}\right) f_{i}(t)-M_{i}\left(q_{i}\right) \dot{f}_{i}(t)\right) \\
& +\frac{1}{2} \dot{M}_{i}\left(q_{i}\right) s_{i}^{2}-\frac{1}{\eta_{i \phi}} \tilde{\theta}_{i \phi}^{T} \hat{\hat{\theta}}_{i \phi}-\frac{1}{\eta_{i M}} \tilde{\theta}_{i M}^{T} \dot{\hat{\theta}}_{i M} \\
& \left.-\frac{1}{\eta_{i C}} \tilde{\theta}_{i C}^{T} \dot{\hat{\theta}}_{i C}-\frac{1}{\eta_{i \delta}} \tilde{\delta}_{i} \dot{\hat{\delta}}_{i}\right] \\
= & \sum_{i=1}^{n}\left[s _ { i } \left(Z_{i}(q, \dot{q}, \ddot{q})+\phi_{i}\left(x_{i}\right)-C_{i}\left(q_{i}, \dot{q}_{i}\right) f_{i}(t)\right.\right. \\
& \left.-M_{i}\left(q_{i}\right) \dot{f}_{i}(t)-u_{i}\right)-\frac{1}{\eta_{i \phi}} \tilde{\theta}_{i \phi}^{T} \dot{\hat{\theta}}_{i \phi}-\frac{1}{\eta_{i M}} \tilde{\theta}_{i M}^{T} \dot{\hat{\theta}}_{i M} \\
& \left.-\frac{1}{\eta_{i C}} \tilde{\theta}_{i C}^{T} \dot{\hat{\theta}}_{i C}-\frac{1}{\eta_{i \delta}} \tilde{\delta}_{i} \dot{\hat{\delta}}_{i}\right]
\end{aligned}
$$

Substituting (17)-(19) and (28) into (39), we have

$$
\begin{aligned}
\dot{V}= & \sum_{i=1}^{n}\left[s _ { i } \left(\theta_{i \phi}^{T} \sigma_{i \phi}-\theta_{i C}^{T} \sigma_{i C} f_{i}(t)-\theta_{i M}^{T} \sigma_{i M} \dot{f}_{i}(t)+\varepsilon_{i 1}\right.\right. \\
& \left.-\varepsilon_{i 2} \dot{f}_{i}(t)-\varepsilon_{i 3} f_{i}(t)-k_{i} s_{i}+Z_{i}(q, \dot{q}, \ddot{q})-u_{i}\right) \\
& \left.-\frac{1}{\eta_{i \phi}} \tilde{\theta}_{i \phi}^{T} \dot{\hat{\theta}}_{i \phi}-\frac{1}{\eta_{i M}} \tilde{\theta}_{i M}^{T} \dot{\hat{\theta}}_{i M}-\frac{1}{\eta_{i C}} \tilde{\theta}_{i C}^{T} \dot{\hat{\theta}}_{i C}-\frac{1}{\eta_{i \delta}} \tilde{\delta}_{i} \dot{\hat{\delta}}_{i}\right] \\
= & \sum_{i=1}^{n}\left[s _ { i } \left(\left(\theta_{i \phi}^{T} \sigma_{i \phi}-\hat{\theta}_{i \phi}^{T} \sigma_{i \phi}\right)-\left(\theta_{i C}^{T} \sigma_{i C}-\hat{\theta}_{i C}^{T} \sigma_{i C}\right) f_{i}(t)\right.\right. \\
& \left.-\left(\theta_{i M}^{T} \sigma_{i M}-\hat{\theta}_{i M}^{T} \sigma_{i M}\right) \dot{f}_{i}(t)+\varepsilon_{i}-k_{i} s_{i}+Z_{i}(q, \dot{q}, \ddot{q})-u_{i c}\right) \\
& \left.-\frac{1}{\eta_{i \phi}} \tilde{\theta}_{i \phi}^{T} \dot{\hat{\theta}}_{i \phi}-\frac{1}{\eta_{i M}} \tilde{\theta}_{i M}^{T} \dot{\hat{\theta}}_{i M}-\frac{1}{\eta_{i C}} \tilde{\theta}_{i C}^{T} \dot{\hat{\theta}}_{i C}-\frac{1}{\eta_{i \delta}} \tilde{\delta}_{i} \dot{\hat{\delta}}_{i}\right] \\
& \leq \sum_{i=1}^{n}\left[s _ { i } \left(\left(\tilde{\theta}_{i \phi}^{T} \sigma_{i \phi}-\frac{1}{\eta_{i \phi}} \tilde{\theta}_{i \phi}^{T} \dot{\hat{\theta}}_{i \phi}\right)-\left(\tilde{\theta}_{i M}^{T} \sigma_{i M} \dot{f}_{i}(t)\right.\right.\right. \\
& \left.-\frac{1}{\eta_{i M}} \tilde{\theta}_{i M}^{T} \dot{\hat{\theta}}_{i M}\right)+\left|\varepsilon_{i}\right|\left|s_{i}\right|-u_{i c}-\left(\tilde{\theta}_{i C}^{T} \sigma_{i C} f_{i}(t)\right. \\
& \left.\left.\left.-\frac{1}{\eta_{i C}} \tilde{\theta}_{i C}^{T} \dot{\hat{\theta}}_{i C}\right)\right)-\frac{1}{\eta_{i \delta}} \tilde{\delta}_{i} \dot{\hat{\delta}}_{i}\right]+\max _{i j}\left\{d_{i j}\right\} \sum_{i=1}^{n}\left|s_{i}\right| \sum_{j=1}^{n} S_{j}
\end{aligned}
$$

It's worth noting that $\left|s_{i}\right| \leq\left|s_{j}\right| \Leftrightarrow\left|S_{i}\right| \leq\left|S_{j}\right|$, and by virtue of Chebyshev inequality, 


$$
\sum_{i=1}^{n}\left|s_{i}\right| \sum_{j=1}^{n} S_{j} \leq n \sum_{i=1}^{n}\left|s_{i}\right| S_{i}
$$

Substituting (29), (34)-(36) and (41) into (40), we have

$$
\begin{aligned}
\dot{V} \leq & \sum_{i=1}^{n}\left[s_{i}\left(-\varepsilon_{0} \operatorname{sgn}\left(s_{i}\right)-\hat{\delta}_{i} \operatorname{sgn}\left(s_{i}\right) S_{i}\right)-k_{i} s_{i}^{2}+\left|\varepsilon_{i}\right|\left|s_{i}\right|\right. \\
& \left.-\frac{1}{\eta_{i \delta}} \tilde{\delta}_{i} \dot{\hat{\delta}}_{i}\right]+n \max _{i j}\left\{d_{i j}\right\} \sum_{i=1}^{n}\left|s_{i}\right| S_{i} \\
\leq & \sum_{i=1}^{n}\left[-k_{i} s_{i}^{2}+\left|s_{i}\right|\left|\varepsilon_{i}\right|-\varepsilon_{0}\left|s_{i}\right|+\delta_{i}\left|s_{i}\right| S_{i}\right. \\
& \left.-\hat{\delta}_{i}\left|s_{i}\right| S_{i}-\frac{1}{\eta_{i \delta}} \tilde{\delta}_{i} \dot{\hat{\delta}}_{i}\right] \\
\leq & \sum_{i=1}^{n}\left[-k_{i} s_{i}^{2}-\left(\varepsilon_{0}-\left|\varepsilon_{i}\right|\right)\left|s_{i}\right|+\tilde{\delta}_{i}\left|s_{i}\right| S_{i}-\frac{1}{\eta_{i \delta}} \tilde{\delta}_{i} \dot{\hat{\delta}}_{i}\right]
\end{aligned}
$$

Then substituting (37) into (42),

$$
\begin{aligned}
\dot{V} & \leq \sum_{i=1}^{n}\left[-k_{i} s_{i}^{2}-\left(\varepsilon_{0}-\left|\varepsilon_{i}\right|\right)\left|s_{i}\right|\right] \\
& \leq \sum_{i=1}^{n}\left(-k_{i} s_{i}^{2}\right)=\Phi(t)
\end{aligned}
$$

Integrating $\Phi(t)$ from 0 to $t$ yields

$$
V(0) \geq V(t)+\int_{0}^{t} \Phi(\tau) d \tau \geq \int_{0}^{t} \Phi(\tau) d \tau
$$

Therefore $0 \leq \int_{0}^{t} \Phi(\tau) d \tau \leq V(0)$. Since $V(0)$ is positive and finite, $\lim _{t \rightarrow \infty} \int_{0}^{t} \Phi(\tau) d \tau$ exists and finite. According to Barbalat Lemma, $\lim _{t \rightarrow \infty} \Phi(t)=0$, it implies that $\lim _{t \rightarrow \infty} s_{i}=0$, namely, the tracking error $e_{i}=q_{i d}-q_{i}$ is convergent to zero asymptotically.

\section{Fault Detection and Isolation}

The aim to fault detection is not only to detect whether a fault occurs or not, but also to determine the time and location of fault occurrence, on which the decision can be made via a significant residual.

\subsection{Fault detection scheme}

Define the output of the first order filter $z_{i}$ as a new state variable

$$
\dot{z}_{i}=-a_{i} z_{i}+b_{i} y_{i}
$$

where $y_{i}$ is the sensor output, and $a_{i}>0, b_{i} \neq 0$.

Substituting the output of (7) into (45), we have

$$
\dot{z}_{i}=-a z_{i}+b x_{i 1}+b f_{i s}
$$

Now, let $\bar{x}_{i}=\left[x_{i 1}, x_{i 2}, x_{i 3}\right]^{T}=\left[q_{i}, \dot{q}_{i}, z_{i}\right]^{T}$, the new state space expression of the subsystem is

$\left\{\begin{array}{l}\dot{\bar{x}}_{i}=\bar{A}_{i} \bar{x}_{i}+\bar{B}_{i}\left[f_{i}\left(q_{i}, \dot{q}_{i}\right)+g_{i}\left(q_{i}\right) u_{i}+h_{i}(q, \dot{q}, \ddot{q})\right]+\bar{E}_{i} f_{i f} \\ \bar{y}_{i}=\bar{C}_{i} \bar{x}_{i}\end{array}\right.$

where

$$
\begin{gathered}
\bar{A}_{i}=\left[\begin{array}{ccc}
0 & 1 & 0 \\
0 & 0 & 0 \\
b_{i} & 0 & -a_{i}
\end{array}\right], \bar{B}_{i}=\left[\begin{array}{l}
0 \\
1 \\
0
\end{array}\right], \bar{C}_{i}=\left[\begin{array}{ll}
0 & 0 \\
1 & 0 \\
0 & 0
\end{array}\right]^{T}, \\
f_{i f}=\left[\begin{array}{c}
f_{i a} \\
f_{i s}
\end{array}\right], \bar{E}_{i}=\left[\begin{array}{ll}
\bar{E}_{i 1} & \bar{E}_{i 2}
\end{array}\right]=\left[\begin{array}{cc}
0 & 0 \\
g_{i}\left(q_{i}\right) & 0 \\
0 & b
\end{array}\right]
\end{gathered}
$$

It is obvious that $\left(\bar{A}_{i}, \bar{B}_{i}\right)$ is controllable, $\left(\bar{A}_{i}, \bar{C}_{i}\right)$ is observable.

Assumption 5: There exists a proper matrix $L_{i}$ satisfies $A_{0 i}=\bar{A}_{i}-L_{i} \bar{C}_{i}$ with the following Riccati function holds since $\left(\bar{A}_{i}, \bar{C}_{i}\right)$ is observable

$$
A_{0 i}^{T} P_{i}+P_{i} A_{0 i}=-I_{i}
$$

where $P_{i}, I_{i}$ are symmetric positive matrices.

Assumption 6: There exists arbitrary matrices $P_{i}$ and $F_{i}$ such that

$$
P_{i} \bar{B}_{i}=\bar{C}_{i}^{T} F_{i}^{T}
$$

where $F_{i}=\left[\begin{array}{ll}F_{i 1} & F_{i 2}\end{array}\right] \in R^{1 \times 2}$.

Define $\hat{\bar{x}}_{i}$ and $\hat{\bar{y}}_{i}$ are the estimated values of $\bar{x}_{i}$ and $\bar{y}_{i}$, respectively, the state error $\bar{e}_{i}=\hat{\bar{x}}_{i}-\bar{x}_{i}$, output error $\bar{e}_{i y}=\hat{\bar{y}}_{i}-\bar{y}_{i}$. The decentralized sliding mode observer (DSMO) based on a radial basis function neural network (RBFNN) can be established as

$$
\left\{\begin{aligned}
\dot{\bar{x}}_{i}= & \bar{A}_{i} \hat{\bar{x}}_{i}+\bar{B}_{i}\left[\hat{f}_{i}\left(\hat{q}_{i}, \dot{\hat{q}}_{i}\right)+\hat{g}_{i}\left(\hat{q}_{i}\right) u_{i}+v_{i}\right. \\
& \left.-\beta_{i} \operatorname{sgn}\left(\bar{e}_{i}^{T} P_{i} \bar{B}_{i}\right)\right]+L_{i}\left(\bar{y}_{i}-\hat{\bar{y}}_{i}\right) \\
\hat{\bar{y}}_{i}= & \bar{C}_{i} \hat{\bar{x}}_{i}
\end{aligned}\right.
$$


where $\beta_{i} \operatorname{sgn}\left(\bar{e}_{i}^{T} P_{i} \bar{B}_{i}\right)$ is the robust term to compensate the approximate error.

The observation error dynamic model derived from (47) and $(50)$ is

$$
\begin{aligned}
\dot{\bar{e}}_{i}= & \left(\bar{A}_{i}-L_{i} \bar{C}_{i}\right) \bar{e}_{i}-\bar{E}_{i} f_{i f}-\bar{B}_{i} \beta_{i} \operatorname{sgn}\left(\bar{e}_{i}^{T} P_{i} \bar{B}_{i}\right) \\
& +\bar{B}_{i}\left[\left(\hat{f}_{i}-f_{i}\right)+\left(\hat{g}_{i}-g_{i}\right) u_{i}+\left(v_{i}-h_{i}\right)\right]
\end{aligned}
$$

where

$$
v_{i}(t)=-\operatorname{sgn}\left(\bar{e}_{i}^{T} P_{i} \bar{B}_{i}\right) \hat{R}_{i}\left(\left|\bar{e}_{i}^{T} P_{i} \bar{B}_{i}\right|, \hat{\theta}_{i p}\right)
$$

is employed to compensate the interconnection term among the subsystems, where $\hat{R}_{i}\left(\left|\bar{e}_{i}^{T} P_{i} \bar{B}_{i}\right|, \hat{\theta}_{i p}\right)=\hat{\theta}_{i p}^{T} \hat{\sigma}_{i p}\left(\left|\bar{e}_{i}^{T} P_{i} \bar{B}_{i}\right|\right)$, $\hat{\theta}_{i p}$ and $\hat{\sigma}_{i p}$ are the estimation of $\theta_{i p}$ and $\sigma_{i p}$, $\tilde{\theta}_{i p}=\hat{\theta}_{i p}-\theta_{i p}$ and $\tilde{\sigma}_{i p}=\hat{\sigma}_{i p}-\sigma_{i p}$ are their estimation errors, respectively. $\theta_{i f}$ and $\theta_{i g}$ are the weights of ideal NNs, respectively. $\sigma_{i(\cdot)}(\cdot)$ is the NN basic function, and the approximate errors $\varepsilon_{i f}$ and $\varepsilon_{i g}$ are norm bounded.

Using the RBFNNs to approximate the uncertainty term $f_{i}\left(q_{i}, \dot{q}_{i}\right)$ and $g_{i}\left(q_{i}\right)$, where the ideal NNs as

$$
\begin{gathered}
f_{i}\left(q_{i}, \dot{q}_{i}\right)=\theta_{i f}^{T} \sigma_{i f}\left(q_{i}, \dot{q}_{i}\right)-\varepsilon_{i f} \\
g_{i}\left(q_{i}\right)=\theta_{i g}^{T} \sigma_{i g}\left(q_{i}\right)-\varepsilon_{i g}
\end{gathered}
$$

$\hat{\theta}_{i f}$ and $\hat{\theta}_{i g}$ denote the estimation of $\theta_{i f}$ and $\theta_{i g}$, the estimated errors are $\tilde{\theta}_{i f}=\hat{\theta}_{i f}-\theta_{i f}$ and $\tilde{\theta}_{i g}=\hat{\theta}_{i g}-\theta_{i g}$, respectively. Then

$$
\begin{gathered}
\hat{f}_{i}\left(\hat{q}_{i}, \dot{\hat{q}}_{i}\right)=\hat{\theta}_{i f}^{T} \hat{\sigma}_{i f}\left(\hat{q}_{i}, \dot{\hat{q}}_{i}\right) \\
\hat{g}_{i}\left(\hat{q}_{i}\right)=\hat{\theta}_{i g}^{T} \hat{\sigma}_{i g}\left(\hat{q}_{i}\right) \\
\tilde{f}_{i}=\hat{f}_{i}\left(\hat{q}_{i}, \dot{\hat{q}}_{i}\right)-f_{i}\left(q_{i}, \dot{q}_{i}\right)=\tilde{\theta}_{i f}^{T} \hat{\sigma}_{i f}\left(\hat{q}_{i}, \dot{\hat{q}}_{i}\right) \\
+\theta_{i f}^{T}\left(\hat{\sigma}_{i f}\left(\hat{q}_{i}, \dot{\hat{q}}_{i}\right)-\sigma_{i f}\left(q_{i}, \dot{q}_{i}\right)\right)+\varepsilon_{i f} \\
\tilde{g}_{i}=\hat{g}_{i}\left(\hat{q}_{i}\right)-g_{i}\left(q_{i}\right)=\tilde{\theta}_{i g}^{T} \hat{\sigma}_{i g}\left(\hat{q}_{i}\right) \\
+\theta_{i g}^{T}\left(\hat{\sigma}_{i g}\left(\hat{q}_{i}\right)-\sigma_{i g}\left(q_{i}\right)\right)+\varepsilon_{i g}
\end{gathered}
$$

Define the approximate error of the NNs as

$$
\begin{gathered}
\omega_{i 1}=\theta_{i f}^{T}\left(\hat{\sigma}_{i f}\left(\hat{q}_{i}, \dot{\hat{q}}_{i}\right)-\sigma_{i f}\left(q_{i}, \dot{q}_{i}\right)\right)+\varepsilon_{i f} \\
+\theta_{i g}^{T}\left(\hat{\sigma}_{i g}\left(\hat{q}_{i}\right)-\sigma_{i g}\left(q_{i}\right)\right) u_{i}+\varepsilon_{i g} u_{i} \\
\omega_{i 2}=R_{i}\left(\left|\bar{e}_{i}^{T} P_{i} \bar{B}_{i}\right|\right)-\theta_{i p}^{T} \hat{\sigma}_{i p}\left(\left|\bar{e}_{i}^{T} P_{i} \bar{B}_{i}\right|\right) \\
\omega_{i}=\left|\omega_{i 1}\right|+\left|\omega_{i 2}\right|
\end{gathered}
$$

where $R_{i}\left(\left|\bar{e}_{i}^{T} P_{i} \bar{B}_{i}\right|\right)=n \max _{i j}\left\{d_{i j}\right\} E_{i}$

Assumption 7: The interconnection term $h_{i}(q, \dot{q}, \ddot{q})$ is bounded as

$$
\left|h_{i}(q, \dot{q}, \ddot{q})\right| \leq \sum_{j=1}^{n} d_{i j} E_{j}
$$

where $d_{i j}>0$ is unknown constant, and define $E_{i}=1+\left|\bar{e}_{i}^{T} P_{i} \bar{B}_{i}\right|+\left|\bar{e}_{i}^{T} P_{i} \bar{B}_{i}\right|^{2}$.

Assumption 8: The approximate error of the NN $\omega_{i}$ is bounded as $\left|\omega_{i}\right| \leq \beta_{i}$ with $\beta_{i}$ is a positive scalar.

The NN weights $\hat{\theta}_{i f}, \hat{\theta}_{i g}$ and $\hat{\theta}_{i p}$ are updated by

$$
\begin{gathered}
\dot{\hat{\theta}}_{i f}=-2 \eta_{i f} \bar{e}_{i}^{T} P_{i} \bar{B}_{i} \hat{\sigma}_{i f} \\
\dot{\hat{\theta}}_{i g}=-2 \eta_{i g} \bar{e}_{i}^{T} P_{i} \bar{B}_{i} \hat{\sigma}_{i g} u_{i} \\
\dot{\hat{\theta}}_{i p}=2 \eta_{i p}\left|\bar{e}_{i}^{T} P_{i} \bar{B}_{i}\right| \hat{\sigma}_{i p}
\end{gathered}
$$

Theorem 2: Consider the subsystem dynamic model (2) with the assumptions 5-8, the observation error (51) obtained by the designed DSMO (50) and the subsystem state space equation (47), as well as the adaptive update laws (63)-(65), the DSMO is asymptotically stable, it implies that $\bar{e}_{i}$ could converge to zero asymptotically.

The proof is similar to section 3 , so it is omitted here.

From (51), we can know that if a fault occurs, $\bar{E}_{i} f_{i f} \neq 0$, and definite change will occur in $\bar{e}_{i}$. Thus, $\bar{e}_{i}$ reflects the occurrence of faults. Summarily, the fault detection scheme can be devised as follows.

Fault detection scheme: Faults can be detected if the residual $\bar{e}_{i}$ exceeds a predefined threshold $\varphi_{i}$. Otherwise, the system is fault-free within the considered time. The detection time $t_{i d}$ is defined as the first moment such that $\bar{e}_{i}$ is greater than $\varphi_{i}$.

\subsection{Fault isolation scheme}

After a fault being detected, to determine its location is next objective, which is referred to fault isolation. In this subsection, based on the DSMO, we present a fault isolation scheme, which could avoid the affection caused by the faulty system to the fault-free subsystems.

Let $e_{i k}(k=s, a)$ describes the observation error. $k=s$ denotes the sensor fault. Define state observe error and the output error as $e_{i s}=\hat{\bar{x}}_{i s}-\bar{x}_{i}$ and $e_{i y s}=\hat{\bar{y}}_{i s}-\bar{y}_{i}$, the designed sensor fault observer is expressed as

$$
\left\{\begin{aligned}
\dot{\hat{\bar{x}}}_{i s}= & \bar{A}_{i} \hat{\bar{x}}_{i s}+\bar{B}_{i}\left[\hat{f}_{i}\left(\hat{q}_{i}, \dot{\hat{q}}_{i}\right)+\hat{g}_{i}\left(\hat{q}_{i}\right) u_{i}+v_{i}\right. \\
& \left.-\beta_{i} \operatorname{sgn}\left(e_{i s}^{T} P_{i} \bar{B}_{i}\right)\right]+\bar{E}_{i s} v_{i s}+L_{i}\left(\bar{y}_{i}-\hat{\bar{y}}_{i s}\right) \\
\hat{\bar{y}}_{i s}= & \bar{C}_{i} \hat{\bar{x}}_{i s}
\end{aligned}\right.
$$


where

$$
v_{i s}=\left\{\begin{array}{c}
-\rho_{i s} \frac{F_{i s} e_{i y s}}{\left\|F_{i s} e_{i y s}\right\|}, \text { if }\left|e_{i y s}\right|>\varsigma \\
0 \text {,if }\left|e_{i y s}\right| \leq \varsigma
\end{array}\right.
$$

where $\rho_{i s}$ is an adjustable constant, $\zeta$ is a sufficiently small positive scalar, thereby the output error is limited within a neighborhood.

Assumption 9: There exists arbitrary matrices $\bar{P}_{i}$ and $F_{i s}$ such that

$$
\bar{P}_{i} \bar{E}_{i s}=\bar{C}_{i}^{T} F_{i s}^{T}
$$

where $F_{i s}=\left[\begin{array}{ll}F_{i s 1} & F_{i s 2}\end{array}\right] \in R^{1 \times 2}$.

Comparing (47) to (66), the observation error dynamic could be expressed as

$$
\begin{aligned}
\dot{e}_{i s}= & \left(\bar{A}_{i}-L_{i} \bar{C}_{i}\right) e_{i s}+\bar{B}_{i}\left[\left(\hat{f}_{i}-f_{i}\right)+\left(\hat{g}_{i}-g_{i}\right) u_{i}+\left(v_{i}-h_{i}\right)\right] \\
& +\bar{E}_{i s} v_{i s}-\bar{E}_{i 1} f_{i a}-\bar{E}_{i 2} f_{i s}-\bar{B}_{i} \beta_{i} \operatorname{sgn}\left(e_{i s}^{T} \bar{P}_{i} \bar{B}_{i}\right)
\end{aligned}
$$

where $v_{i}\left(\left|e_{i s}^{T} \bar{P}_{i} \bar{B}_{i}\right|, \hat{\theta}_{i P}\right)$ is utilized to compensate the interconnection, the RBFNNs $\hat{f}_{i}\left(\hat{q}_{i}, \dot{\hat{q}}_{i}, \hat{\theta}_{i f}\right)$ and $\hat{g}_{i}\left(\hat{q}_{i}, \hat{\theta}_{i g}\right)$ approximate the unknown terms $f_{i}\left(q_{i}, \dot{q}_{i}\right)$ and $g_{i}\left(q_{i}\right)$, $\beta_{i} \operatorname{sgn}\left(e_{i s}^{T} \bar{P}_{i} \bar{B}_{i}\right)$ is a robust term, which could offset the approximation error.

$k=a$ denotes the actuator fault. Define the state observation error as $e_{i a}=\bar{x}_{i}-\hat{\bar{x}}_{i a}$, output error as $e_{i a s}=\bar{y}_{i}-\hat{\bar{y}}_{i a}$, the designed actuator fault observer is described as

$$
\left\{\begin{aligned}
\dot{\bar{x}}_{i a 1}= & \hat{\bar{x}}_{i a 2}+K_{i 1} e_{i a 1} \\
\dot{\bar{x}}_{i a 2}= & \hat{f}_{i}\left(\hat{q}_{i}, \dot{\hat{q}}_{i}, \hat{\theta}_{i f}\right)+\hat{g}_{i}\left(\hat{q}_{i}, \hat{\theta}_{i g}\right) u_{i} \\
& +v_{i}\left(e_{i a 2}, \hat{\theta}_{i P}\right)+K_{i 2} e_{i a 2} \\
\dot{\bar{x}}_{i a 3}= & b_{i} \hat{\bar{x}}_{i a 1}-a_{i} \hat{\bar{x}}_{i a 3}+b_{i} v_{i a}+K_{i 3} e_{i a 3}
\end{aligned}\right.
$$

where

$$
v_{i a}=\left\{\begin{array}{c}
-\gamma_{i} \frac{e_{i a 3}}{\left\|e_{i a 3}\right\|}, \text { if }\left\|e_{i a 3}\right\|>\varsigma \\
0, \text { if }\left\|e_{i a 3}\right\| \leq \varsigma
\end{array}\right.
$$

where $\gamma_{i}$ is an adjustable scalar, $\varsigma$ is still a sufficiently small positive constant.

Comparing (47) to (70), the dynamic observation error is

$$
\left\{\begin{array}{l}
\dot{e}_{i a 1}=e_{i a 2}-K_{i 1} e_{i a 1} \\
\dot{e}_{i a 2}=\left(f_{i}-\hat{f}_{i}\right)+\left(g_{i}-\hat{g}_{i}\right) u_{i}+h_{i}-v_{i}+g_{i} f_{i a}-K_{i 2} e_{i a 2} \\
\dot{e}_{i a 3}=b_{i} e_{i a 1}-a_{i} e_{i a 3}+b_{i} f_{i s}-b_{i} v_{i a}-K_{i 3} e_{i a 3}
\end{array}\right.
$$

Theorem 3: Consider a bank of observers (66) and (70), the observation error dynamic model as (69) and (72), and the assumptions 5-9 with the adaptive laws (63)-(65), the observation errors can converge to zero. In other words, the estimated state can follow to the actual state $\bar{x}_{i}$. Once the subsystem is faulty, $e_{i k}$ could definitely change.

Proof: When $k=s$, choose Lyapunov candidate as

$$
\begin{aligned}
V_{s} & =\sum_{i=1}^{n} V_{i s} \\
& =\sum_{i=1}^{n}\left[e_{i s}^{T} P_{i} e_{i s}+\frac{1}{2 \eta_{i f}} \tilde{\theta}_{i f}^{T} \tilde{\theta}_{i f}+\frac{1}{2 \eta_{i g}} \tilde{\theta}_{i g}^{T} \tilde{\theta}_{i g}+\frac{1}{2 \eta_{i p}} \tilde{\theta}_{i p}^{T} \tilde{\theta}_{i p}\right]
\end{aligned}
$$

Its time derivative

$$
\begin{aligned}
\dot{V}_{s}= & \sum_{i=1}^{n}\left[\dot{e}_{i s}^{T} P_{i} e_{i s}+e_{i a}^{T} P_{i} \dot{e}_{i s}+\frac{1}{\eta_{i f}} \tilde{\theta}_{i f}^{T} \dot{\hat{\theta}}_{i f}\right. \\
& \left.+\frac{1}{\eta_{i g}} \tilde{\theta}_{i g}^{T} \dot{\hat{\theta}}_{i g}+\frac{1}{\eta_{i p}} \tilde{\theta}_{i p}^{T} \dot{\hat{\theta}}_{i p}\right]
\end{aligned}
$$

Substituting (47) and (66) into (74), one can obtain that

$$
\begin{aligned}
\dot{V}_{s}= & \sum_{i=1}^{n}\left[e_{i s}^{T}\left(A_{0 i}^{T} P_{i}+\bar{P}_{i} A_{0 i}\right) e_{i s}+2 e_{i s}^{T} P_{i} \bar{B}_{i}\left(\tilde{f}_{i}+\tilde{g}_{i} u_{i}\right)\right. \\
& -2 \beta_{i}\left|e_{i s}^{T} P_{i} \bar{B}_{i}\right|+\frac{1}{2 \eta_{i f}} \tilde{\theta}_{i f}^{T} \dot{\hat{\theta}}_{i f}+\frac{1}{2 \eta_{i g}} \tilde{\theta}_{i g}^{T} \dot{\hat{\theta}}_{i g}+\frac{1}{\eta_{i p}} \tilde{\theta}_{i p}^{T} \dot{\hat{\theta}}_{i p} \\
& \left.+2 e_{i s}^{T} P_{i} \bar{B}_{i}\left(v_{i}-h_{i}\right)\right]+\sum_{i=1}^{n}\left[2 e_{i s}^{T} P_{i} \bar{E}_{i}\left(v_{i s}-f_{i a}\right)\right]
\end{aligned}
$$

According to similar proof in section 2, we have

$$
\begin{aligned}
\dot{V}_{s} & \leq \sum_{i=1}^{n}\left[-\lambda_{\min }\left(Q_{i}\right)\left|e_{i s}\right|^{2}+2 e_{i s}^{T} P_{i} \bar{E}_{i s}\left(v_{i s}-f_{i a}\right)\right] \\
& \leq \sum_{i=1}^{n}\left[-\lambda_{\min }\left(Q_{i}\right)\left|e_{i s}\right|^{2}-2\left\|F_{i s} e_{i y s}\right\|\left(\rho_{i}-\alpha_{i 1}\right)\right] \\
& \leq \sum_{i=1}^{n}\left[-\lambda_{\min }\left(Q_{i}\right)\left|e_{i s}\right|^{2}\right]
\end{aligned}
$$

It is clear that $V(t) \leq V(0)$, according to Barbalat Lemma, $\lim _{\mathrm{t} \rightarrow \infty} e_{i s}=0$, which means $e_{i s}$ converge to zero. When $k=a$, choose the Lyapunov candidate function as 


$$
V_{a}=V_{a 1}+V_{a 2}+V_{a 3}
$$

where

$$
\begin{aligned}
V_{a 1} & =\sum_{i=1}^{n}\left(\frac{1}{2} e_{i a 1}^{2}\right) \\
V_{a 2} & =\sum_{i=1}^{n}\left(\frac{1}{2} e_{i a 2}^{2}+\frac{1}{2 \eta_{i f}} \tilde{\theta}_{i f}^{2}+\frac{1}{2 \eta_{i g}} \tilde{\theta}_{i g}^{2}+\frac{1}{2 \eta_{i p}} \tilde{\theta}_{i p}^{2}\right) \\
V_{a 3} & =\sum_{i=1}^{n}\left(\frac{1}{2} e_{i a 3}^{2}\right)
\end{aligned}
$$

(1) The time derivative of $V_{a 3}$ is

$$
\begin{aligned}
\dot{V}_{a 3}= & \sum_{i=1}^{n}\left(e_{i a 3} \dot{e}_{i a 3}\right) \\
\leq & \sum_{i=1}^{n}\left(-\left(a_{i}+K_{i 3}\right) e_{i a 3}^{2}+b_{i}\left|e_{i a 1}\right|\left|e_{i a 3}\right|+b_{i} \alpha_{i 2}\left|e_{i a 3}\right|\right. \\
& \left.-b_{i} \gamma_{i} e_{i a 3} \operatorname{sgn}\left(e_{i a 3}\right)\right) \\
\leq & \sum_{i=1}^{n}\left(-\left|e_{i a 3}\right|\left(\left(a_{i}+K_{i 3}\right)\left|e_{i a 3}\right|-b_{i}\left|e_{i a 1}\right|\right)\right. \\
& \left.\quad-b_{i}\left|e_{i a 3}\right|\left(\gamma_{i}-\alpha_{i 2}\right)\right)
\end{aligned}
$$

$\dot{V}_{a 3} \leq 0$ if $K_{i 3}$ and $\gamma_{i}$ satisfy $\left(a_{i}+K_{i 3}\right)\left|e_{i a 3}\right|-b_{i}\left|e_{i a 1}\right|>0$ and $\gamma_{i}-\alpha_{i 2}>0$ at the same time. According to the sliding mode equivalent principle,

$$
\left(\left(\gamma_{i}-\alpha_{i 2}\right) \operatorname{sgn}\left(e_{i a 3}\right)\right)_{e q}=e_{i a 1}
$$

where $\left(\gamma_{i}-\alpha_{i 2}\right) \operatorname{sgn}\left(e_{i a 3}\right)$ is the discontinuous equivalent output injection value.

(2) The time derivative of $V_{a 1}$ is

$$
\begin{aligned}
\dot{V}_{a 1} & =\sum_{i=1}^{n}\left(e_{i a 1} \dot{e}_{i a 1}\right) \\
& =\sum_{i=1}^{n}\left(e_{i a 1}\left(e_{i a 2}-K_{i 1} e_{i a 1}\right)\right) \\
& \leq \sum_{i=1}^{n}\left(-\left|e_{i a 1}\right|\left(K_{i 1}\left|e_{i a 1}\right|-\left|e_{i a 2}\right|\right)\right)
\end{aligned}
$$

$\dot{V}_{a 1} \leq 0$ if the selected $K_{i 1}$ satisfies $K_{i 1}\left|e_{i a 1}\right|-\left|e_{i a 2}\right|>0$.

(3) The time derivative of $V_{a 2}$ is

$$
\dot{V}_{a 2}=\sum_{i=1}^{n}\left[e_{i a 2}\left(\left(f_{i}-\hat{f}_{i}\right)+\left(g_{i}-\hat{g}_{i}\right) u_{i}+h_{i}-v_{i}-K_{i 2} e_{i a 2}\right)\right.
$$

$$
\begin{aligned}
\left.-\frac{1}{\eta_{i f}} \tilde{\theta}_{i f} \dot{\hat{\theta}}_{i f}-\frac{1}{\eta_{i g}} \tilde{\theta}_{i g} \dot{\hat{\theta}}_{i g}-\frac{1}{\eta_{i p}} \tilde{\theta}_{i p} \dot{\hat{\theta}}_{i p}\right] \\
\leq \sum_{i=1}^{n}\left[\left(e_{i a 2} \tilde{\theta}_{i f} \hat{\sigma}_{i f}-\frac{1}{\eta_{i f}} \tilde{\theta}_{i f} \dot{\hat{\theta}}_{i f}\right)+\left(e_{i a 2} \tilde{\theta}_{i g} \hat{\sigma}_{i g} u_{i}\right.\right. \\
\left.\quad-\frac{1}{\eta_{i g}} \tilde{\theta}_{i g} \dot{\hat{\theta}}_{i g}\right)+\left|e_{i a 2}\right|\left|\omega_{i 1}\right|+\left|e_{i a 2}\right|\left|h_{i}\right|-\left|e_{i a 2}\right| \hat{\theta}_{i p} \hat{\sigma}_{i p} \\
\left.\quad-K_{i 2}\left|e_{i a 2}\right|^{2}-\frac{1}{\eta_{i p}} \tilde{\theta}_{i p} \dot{\hat{\theta}}_{i p}\right] \\
\leq \sum_{i=1}^{n}\left[-\left|e_{i a 2}\right| \hat{\theta}_{i p} \hat{\sigma}_{i p}-K_{i 2}\left|e_{i a 2}\right|^{2}+\left|e_{i a 2}\right|\left|\omega_{i 1}\right|\right. \\
\left.\quad-\frac{1}{\eta_{i p}} \tilde{\theta}_{i p} \dot{\hat{\theta}}_{i p}\right]+\max _{i j}\left\{d_{i j}\right\} \sum_{i=1}^{n}\left|e_{i a 2}\right| \sum_{j=1}^{n} E_{j}
\end{aligned}
$$

Noticing that $\left|e_{i a 2}\right| \leq\left|e_{j a 2}\right| \Leftrightarrow E_{i} \leq E_{j}$, by virtue of Chebyshev inequality,

$$
\sum_{i=1}^{n}\left|e_{i a 2}\right| \sum_{j=1}^{n} E_{j} \leq n \sum_{i=1}^{n}\left|e_{i a 2}\right| E_{i}
$$

Substituting (85) into (84), we have

$$
\begin{aligned}
\dot{V}_{a 2} \leq & \sum_{i=1}^{n}\left[\left|e_{i a 2}\right|\left(n \max \left\{d_{i j}\right\} E_{i j}-\hat{\theta}_{i p} \hat{\sigma}_{i p}\right)+\left|e_{i a 2}\right|\left|\omega_{i 1}\right|\right. \\
& \left.-K_{i 2}\left|e_{i a 2}\right|^{2}-\frac{1}{\eta_{i p}} \tilde{\theta}_{i p} \dot{\hat{\theta}}_{i p}\right] \\
\leq & \sum_{i=1}^{n}\left[\left(\left|e_{i a 2}\right| \tilde{\theta}_{i p} \hat{\sigma}_{i p}-\frac{1}{\eta_{i p}} \tilde{\theta}_{i p} \dot{\hat{\theta}}_{i p}\right)+\left|e_{i a 2}\right|\left|\omega_{i 1}\right|\right. \\
& \left.+\left|e_{i a 2}\right|\left|\omega_{i 2}\right|-K_{i 2}\left|e_{i a 2}\right|^{2}\right] \\
\leq & \sum_{i=1}^{n}\left[\left|e_{i a 2}\right|\left(\left|\omega_{i}\right|-K_{i 2}\left|e_{i a 2}\right|\right)\right]
\end{aligned}
$$

$\dot{V}_{a 2} \leq 0$ if the selected $K_{i 2}$ satisfies $K_{i 2}\left|e_{i a 2}\right|-\left|\omega_{i}\right|>0$. From the analysis above, it is clear $V(t) \leq V(0)$, thus $e_{i a}$ is bounded. According to Barbalat Lemma, $\lim _{\mathrm{t} \rightarrow \infty} e_{i a}=0$ which means $e_{i a}$ can converge to zero.

\section{Numerical simulation}

In order to verify the effectiveness of the proposed methods, two 3-DOF reconfigurable manipulators with different configurations shown as Fig. 1 are adopted for numerical simulation. 


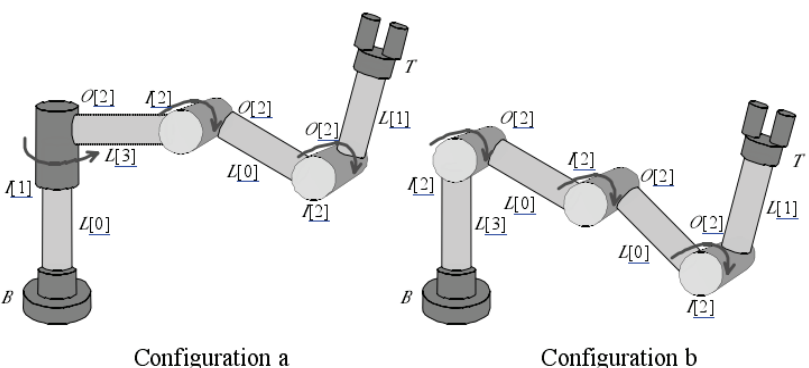

Fig.1. Reconfigurable manipulators for simulation

The friction terms of configuration $a$ and configuration $b$ are respectively expressed as follows:

Configuration a:

$$
F(q, \dot{q})=\left[\begin{array}{c}
\dot{q}_{1}+10 \sin \left(3 q_{1}\right)+2 \operatorname{sgn}\left(\dot{q}_{1}\right) \\
1.2 \dot{q}_{2}+5 \sin \left(2 q_{2}\right)+\operatorname{sgn}\left(\dot{q}_{2}\right) \\
0.6 \dot{q}_{3}+7 \sin \left(4 q_{3}\right)+\operatorname{sgn}\left(\dot{q}_{3}\right)
\end{array}\right]
$$

Configuration $\mathrm{b}$ :

$$
F(q, \dot{q})=\left[\begin{array}{c}
2 \dot{q}_{1}+5 \sin \left(2 q_{1}\right)+\operatorname{sgn}\left(\dot{q}_{1}\right) \\
1.5 \dot{q}_{2}+5 \sin \left(q_{2}\right)+1.2 \operatorname{sgn}\left(\dot{q}_{2}\right) \\
1.8 \dot{q}_{3}+5 \sin \left(3 q_{3}\right)+\operatorname{sgn}\left(\dot{q}_{3}\right)
\end{array}\right]
$$

The joint desired trajectories of configuration $a$ are

$$
\begin{aligned}
& q_{1 d}=0.5 \cos (t)+0.2 \sin (3 t) \\
& q_{2 d}=-0.2 \sin (t)+0.4 \cos (2 t) \\
& q_{3 d}=0.3 \cos (3 t)-0.5 \sin (2 t)
\end{aligned}
$$

The joint desired trajectories of configuration $b$ are

$$
\begin{aligned}
& q_{1 d}=0.3 \cos (2 t)+0.1 \sin (t) \\
& q_{2 d}=0.2 \sin (3 t)+0.3 \cos (2 t) \\
& q_{3 d}=0.5 \sin (2 t)+0.3 \cos (t)
\end{aligned}
$$

The initial positions and velocities of each joint are $q_{1}(0)=q_{2}(0)=q_{3}(0)=1$ and $\dot{q}_{1}(0)=\dot{q}_{2}(0)=\dot{q}_{3}(0)=0$.

\subsection{Simulation for decentralized control scheme}

In this subsection, the proposed decentralized controller (27) is applied to the reconfigurable manipulator in faultfree. The initial values of $\hat{\theta}_{i \phi}, \hat{\theta}_{i M}, \hat{\theta}_{i C}$ and $\hat{\delta}_{i}$ are zeros. The control parameters are $\eta_{i \phi}=\eta_{i \delta}=0.02, \varepsilon_{0}=0.01$, $\eta_{i M}=\eta_{i C}=0.01, k_{i}=5, \lambda_{i}=25, f_{i}(t)=f_{i}(0) e^{-20 t}$ with $f_{i}(0)=\dot{q}_{i d}(0)-\dot{q}_{i}(0)+\lambda_{i}\left(q_{i d}(0)-q_{i}(0)\right)$.

From Fig. 2, one can see that the actual trajectories take about only $0.2 s$ to follow their desired trajectories, which means each joint in configuration $a$ obtained good tracking performance.

To further test the effectiveness of the proposed decentralized controller, configuration $b$ is employed for
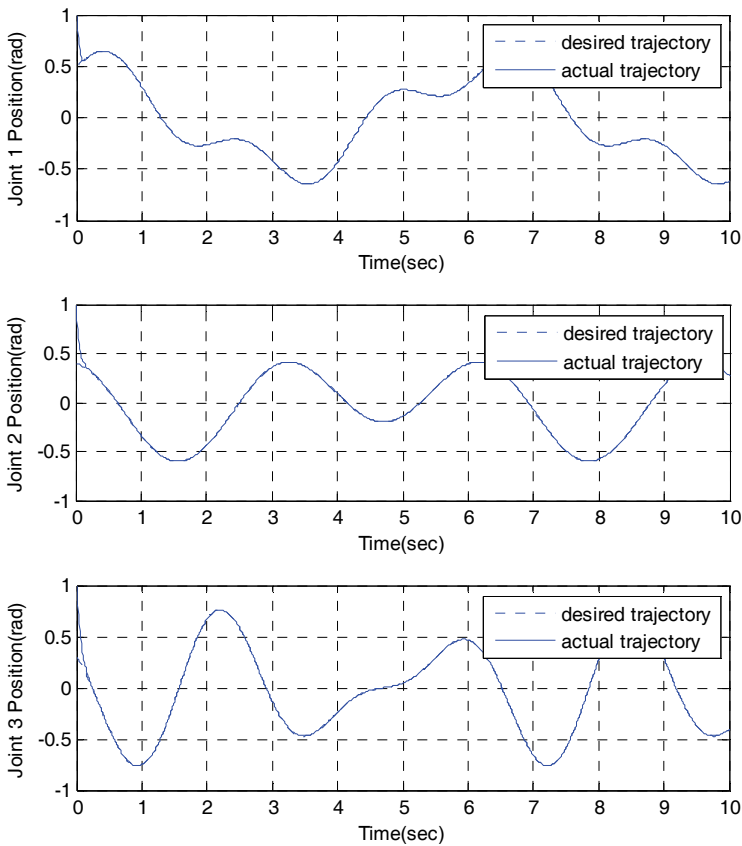

Fig. 2. Joint tracking curves of configuration $a$
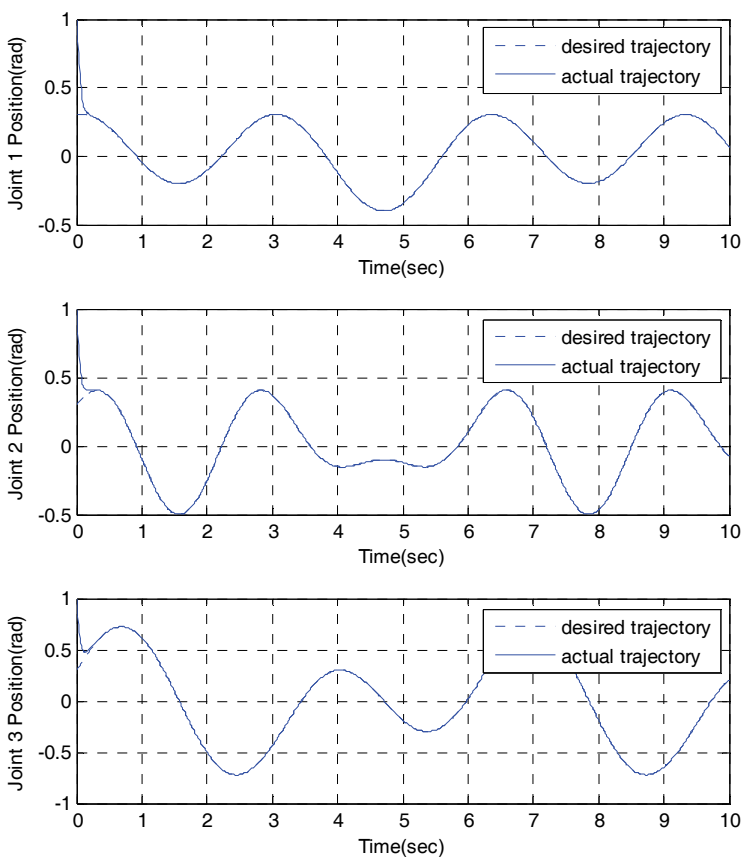

Fig. 3. Joint tracking curves of configuration $b$

simulation with the same control law, and the Fig. 3 shows excellent tracking performance as well. It demonstrates that the proposed control scheme is feasible to different configurations without any control parameters modification.

\subsection{Simulation for FDI}

\subsubsection{Simulation results of fault detection}

In this subsection, the predefined threshold of observation error is $\varphi_{i}=0.03$, the observer gains are $L_{i 1}=2000$, 
$L_{i 2}=1000$ and $L_{i 3}=30$, adaptive gains are $\eta_{i f}=0.02$, $\eta_{i g}=0.01$ and $\eta_{i p}=0.01$, the robust coefficient is $\beta_{i}=0.01$, the filter coefficients are set as $a_{i}=1, b_{i}=1$, respectively.

For the i-th subsystem of reconfiguration manipulators, the RBFNNs are utilized to approximate the terms $f_{i}\left(q_{i}, \dot{q}_{i}\right), g_{i}\left(q_{i}\right)$ and $R_{i}\left(\left|\bar{e}_{i}^{T} P_{i} \bar{B}_{i}\right|\right)$. Where $X=\left[x_{1}, \cdots, x_{n}\right]^{T}$ is the input vector of NN, $\sigma=\left[\sigma_{1}, \cdots, \sigma_{m}\right]^{T}$ is the radial basis function, and the $\mathrm{NN}$ is expressed as

$$
\sigma_{j}=\exp \left(-\left\|X-c_{j}\right\|^{2} / 2 b_{j}^{2}\right)
$$

where $j=1,2, \ldots, m$ denotes the number of nodes in the hidden layer, $c_{j}$ and $b_{j}$ are the central and the width of RBFNN's $j$-th node,

$$
c_{j}=\left[\xi_{1}^{T}, \cdots, \xi_{n}^{T}\right]^{T}, b_{j}=10
$$

where $i=1,2, \cdots, n$ denotes the number of hidden layers in $\mathrm{NN}$, and $\xi_{i}^{T}=\left[\begin{array}{lllllll}-1.5 & -1 & -0.5 & 0 & 0.5 & 1 & 1.5\end{array}\right]^{T}$.

The injected faults are defined as

$$
\left\{\begin{array}{l}
f_{1 a}=10 \sin \left(2 q_{1}\right) \dot{q}_{1}, t \geq 3 \\
f_{2 s}=-0.5 q_{2}, t \geq 5
\end{array}\right.
$$

The observation error curves of configuration $a$ and $b$ shown as Fig. 4 and Fig. 5, respectively, the red lines present the given thresholds. In these figures, from $\mathrm{t}=3 \mathrm{~s}$ and $\mathrm{t}=5 \mathrm{~s}$, the actuator fault $f_{1 a}$ and sensor fault $f_{2 s}$ are embedded on joint 1 and joint 2, respectively. One can observe that the observation errors exceed the thresholds
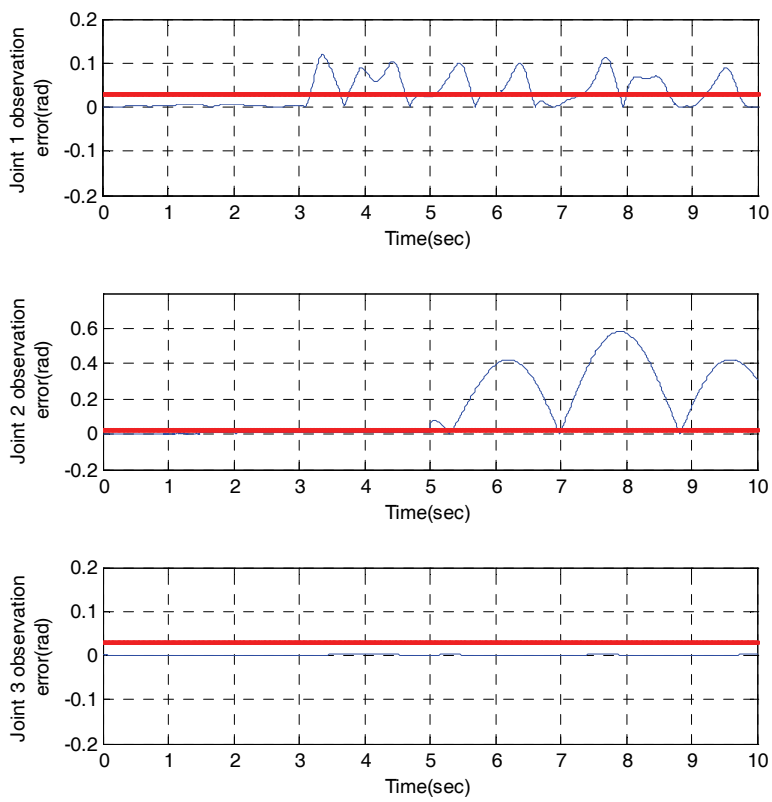

Fig. 4. Observation performance of configuration $a$ in faulty once the fault occurs. Therefore, this proposed scheme can detect the fault in real time.
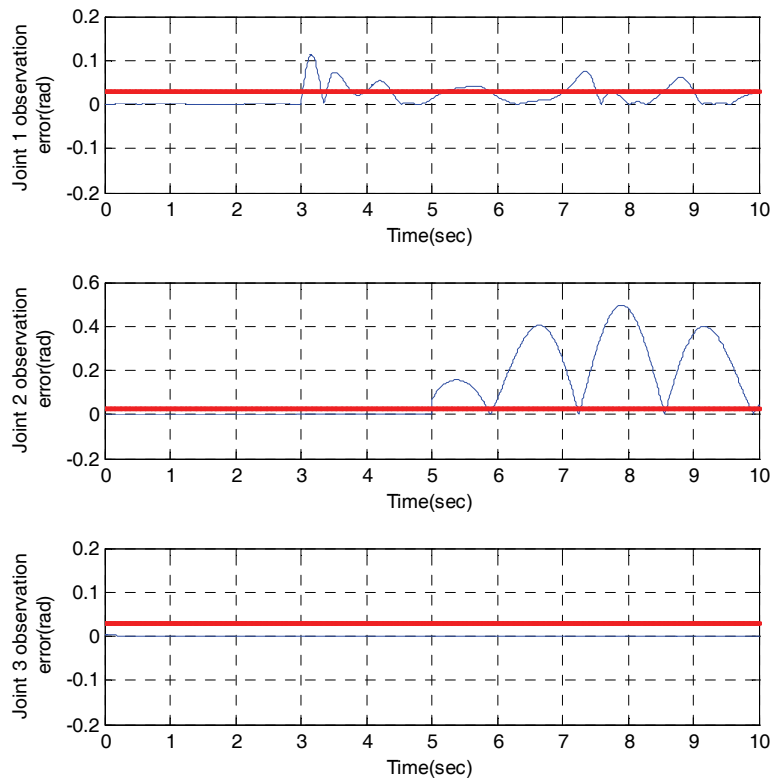

Fig. 5. Observation performance of configuration $b$ in faulty
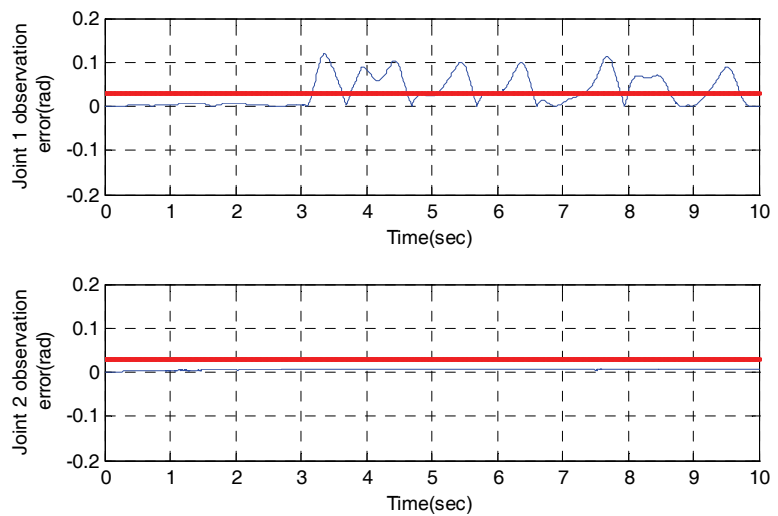

(a) Actuator observer output error curves of configuration $a$
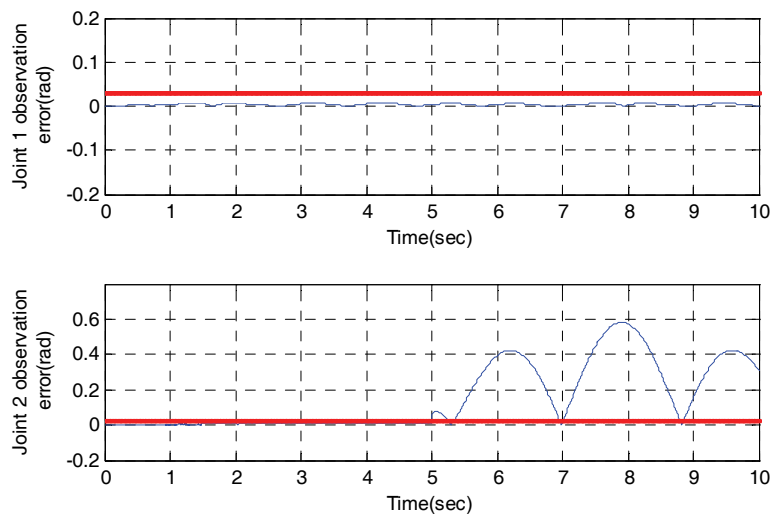

(b) Sensor observer output error curves of configuration $a$

Fig. 6. The bank of observers' output error curves of configuration $a$ 

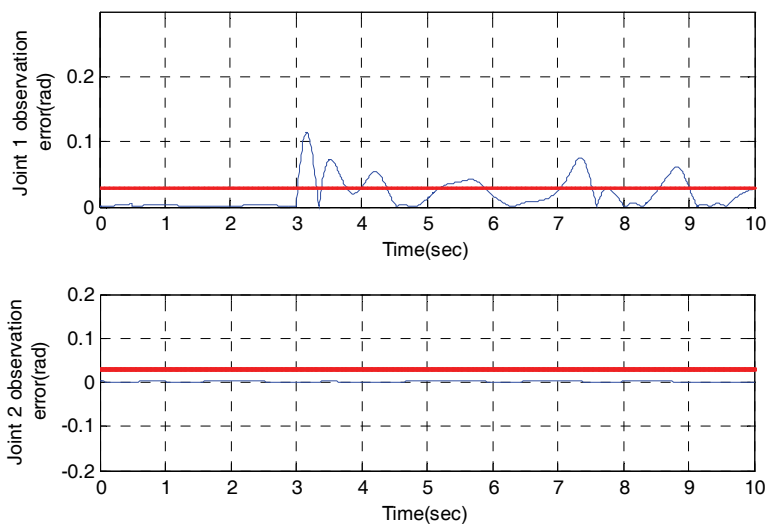

(a) Actuator observer output error curves of configuration $b$
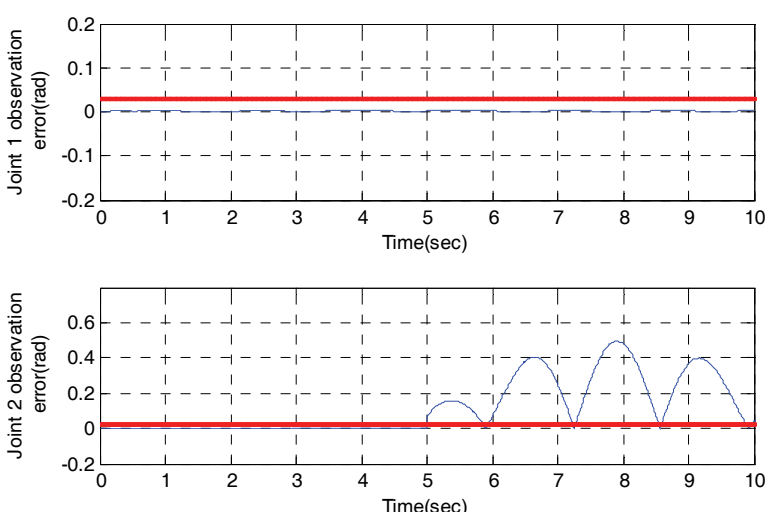

(b) Sensor observer output error curves of configuration $b$

Fig. 7. The bank of observers' output error curves of configuration $b$

\subsubsection{Simulation results of fault isolation}

In this subsection, utilize DSMOs (66) and (70), the actuator fault observer gains are set as $k_{i 1}=100, k_{i 2}=300$, $k_{i 3}=20$. The adjustable sliding mode parameters are $\rho_{i}=130, \gamma_{i}=800$, the sensor observer parameters and the fault are as in section 5.2.1.

Fig. 6(a) shows the actuator observer output curves, one can see that the observation error of joint 1 of configuration $a$ exceeds the predefined threshold after $\mathrm{t}=3 \mathrm{~s}$, and that of joint 2 lies within the given threshold, it proofs that the actuator fault can be isolated from sensor fault. Meanwhile, Fig. 6(b) shows that of sensor fault, the same conclusion can be obtained. To further test the proposed isolation scheme, the configuration $b$ is employed for simulation, whose results shown as Fig. 7. It's worth noting that joint 3 is fault free according to the results of fault detection in the previous subsection, therefore, its simulation result can be omitted here.

\section{Conclusions}

By virtue of DSMO, a multiple faults detection and isolation schemes for reconfigurable manipulator are presented in this paper. Based on the modularization property and Lyapunov stability theory, the adaptive fuzzy decentralized controller is applied to the reconfigurable manipulator in fault-free. Then the multiple faults including actuator fault and sensor fault can be detected by means of the observation errors whether exceed the predefined thresholds or not. After being detected, multiple faults are isolated to decide which joint and which component is faulty. Its effectiveness has been demonstrated by the simulation of two 3-DOF reconfigurable manipulators with different configurations in the presence of multiple faults.

\section{Acknowledgements}

This work was supported by the National Natural Science Foundation of China (61374051) and the Scientific and Technological Development Plan Project in Jilin Province of China (20150520112JH).

\section{References}

[1] Paredis CJJ, Brown HB and Khosla PK., "A rapidly deployable manipulator system," Robot Autonomous Systems, vol. 21, no. 3, pp. 289-304, 1997.

[2] Melek, W.W. and Goldenberg, A.A., "Neurofuzzy control of modular and reconfigurable robots," IEEE/ ASME transactions on mechatronics, vol. 8, no. 3, pp. 381-389, 2003.

[3] Biglarbegian, M., Melek, W.W. and Mendel J.M., "Design of Novel Interval Type-2 Fuzzy Controllers for Modular and Reconfigurable Robots: Theory and Experiments," IEEE Transactions on Industrial Electronics, vol. 58, no. 4, pp. 1371-1384, 2011.

[4] Melek, W.W. and Najjaran, H., "Study of the effect of external disturbances on the position control of IRIS modular and reconfigurable manipulator," in the Proceeding of 2005 IEEE International Conference on Mechatronics Automation, pp. 144-147, 2005.

[5] Biglarbegian, M., Melek, W. W., Mendel, J. M., “On the stability of interval Type-2 TSK fuzzy logic control systems," IEEE Transactions on systems man and Cybernetics part B:Cybernetics, vol. 40, no. 3, pp. 798-818, 2010.

[6] Liu, G., Abdul, S. and Goldenberg, A.A., "Distributed control of modular and reconfigurable robot with torque sensing," Robotica, vol. 26 , no. 1, pp. 75-84, 2008.

[7] Terada, Y. and Murata, S., "Automatic modular assembly and its distributed control," International Journal of Robotics Research, vol. 27, no. 3-4, pp. 445-462, 2008.

[8] Li, Z., Melek, W.W. and Clark, C., "Decentralized robust control of robot manipulators with harmonic drive transmission and application to modular and 
reconfigurable serial arms," Robotica, vol. 27 , no. 2 , pp. 291-302, 2009.

[9] Zhu, M. and Li, Y., "Decentralized adaptive fuzzy sliding mode control for reconfigurable modular manipulators," International Journal of Robust and Nonlinear Control, vol.20, no.4, pp.472-488, 2010.

[10] Huang, S.T.C., Davison E.J. and Kwong, R.H., "Decentralized robust servomechanism problem for large flexible space structures under sensor and actuator failures," IEEE Transactions on Automatic Control, vol.57, no.12, pp.3219-3224, 2012.

[11] Kawakita, M., Yubai, K. and Hirai, J., "The multirate sampling control for a reconfigurable robot," in the Proceeding of $201111^{\text {th }}$ International Conference on Control, Automation and Systems, pp.516-521, 2011.

[12] Lau, H.Y.K., Ko, A. and Lau, T.L., “A decentralized control framework for modular robots," in the Proceedings of 2004 IEEE/RSJ International conference on intelligent robots and systems, pp. 1774-1779, 2004.

[13] Alwi, H, Edwards, C. and Tan, C.P., "Sliding mode estimation schemes for incipient sensor faults," Automatica, vol. 45, no. 7, pp. 1679-1685, 2009.

[14] Rolf Isermann, "Model-based fault-detection and diagnosis-status and applications," Annual Reviews in Control, vol. 29, no. 1, pp. 71-85, 2005.

[15] Izumikawa, Y., Yubai, K. and Hirai, J., "Faulttolerant control system of flexible arm for sensor fault by using reaction force observer," IEEE/ASME Transactions on Mechatronics, vol.10, no.4, pp.391$396,2005$.

[16] Gao, B., Jiang, B., Qi, R. and Xu, Y., "Robust reliable control for a near space vehicle with parametric uncertainties and actuator faults," International Journal of Systems Science, vol. 42, no. 12, pp. 2113-2124, 2011.

[17] P. C. Deng, W. H. Gui and Y. F. Xie, "Latent space transformation based on principal component analysis for adaptive fault detection," IET Control Theory and Applications, vol.4, no.11, pp. 2527-2538, 2010.

[18] Shengfa Yuan and Fulei Chu, "Fault diagnosis based on support vector machines with parameter optimization by artificial immunization algorithm," Mechanical Systems and Signal Processing, vol. 21, no. 3, pp. 1318-1330, 2007.

[19] Anders Lyhne Christensen, Rehan O'Grady, Mauro Birattari and Marco Dorigo, "Fault detection in autonomous robots based on fault injection and learning," Autonomous Robot, vol.24, no.1, pp. 49-67, 2008.

[20] Tolga Yuksel, Abdullah Sezgin, "Two fault detection and isolation schemes for robot manipulators using soft computing techniques," Applied Soft Computing, vol.10, no.1, pp. 125-134, 2010.

[21] Yuan, J., Liu, G.J. and Wu, B., "Power Efficiency Estimation-Based Health Monitoring and Fault
Detection of Modular and Reconfigurable Robot", IEEE Transactions on industrial electronics, vol.58, no.10, pp.4880-4887, 2011.

[22] Ahmad, S., Zhang, H. and Liu, G., "Distributed fault detection for modular and reconfigurable robots with joint torque sensing: a prediction error based approach," Mechatronics, vol. 23, no. 6, pp. 607-616, 2013.

[23] Yuan, S., and Liu, X., "Fault estimator design for a class of switched systems with time-varying delay," International Journal of Systems Science, vol. 42, no. 12, pp. 2125-2135, 2011.

[24] Bo Zhao and Yuanchun Li. "Local joint information based active fault tolerant control for reconfigurable manipulator," Nonlinear Dynamics, vol. 77, no. 3, pp. 859-876, 2014.

[25] C. P. Tan and C. Edwards, "Sliding mode observers for detection and reconstruction of sensor faults," Automatica, vol. 38, no. 10, pp. 1815-1821, 2002.

[26] Qing Wu, Mehrdad Saif, "Robust fault diagnosis of a satellite system using a learning strategy and second order sliding mode observer," IEEE Systems Journal, vol. 4, no. 1, pp. 112-121, 2010.

[27] Xing-Gang Yan and Christopher Edwards, "Sensor fault detection and isolation for nonlinear systems based on a sliding mode observer", International Journal of Adaptive Control and Signal Processing, vol. 21, no. 8-9, pp. 657-673, 2007.

[28] J. Zhang, A.K. Swain and S.K.Nguang, "Detection and isolation of incipient sensor faults for a class of uncertain non-linear systems," IET Control Theory and Applications, vol. 6, no. 12, pp. 1870-1880, 2012

[29] Coradini, M.L., Orlando, G., "Linear unstable plants with saturating actuators: Robust stabilization by a time varying sliding surface," Automatica, vol. 43, no. 1, pp. 88-94, 2007.

[30] Karbasi, H., Huissoon, J. P. and Khajepour, A., "Blend of independent joint control and variable structure systems for uni-drive modular robots," Robotica, vol. 28, no. 1, pp. 149-159, 2010.

[31] Zhao, B. and Li, Y.C., "Multisensor fault identification scheme based on decentralized sliding mode observers applied to reconfigurable manipulators," Mathematical Problems in Engineering, http://dx.doi.org/10.1155/ 2013/327916, 2013.

[32] Efimov, D. and Fridman, L., "Global sliding-mode observer with adjusted gains for locally Lipschitz systems," Automatica, vol. 47, no. 3, pp. 565-570, 2011.

[33] Liu, L., Han, Z. and Li, W., "Global sliding mode control and application in chaotic systems", Nonlinear Dynamics, vol. 56, no. 1-2, pp. 193-198, 2009. 


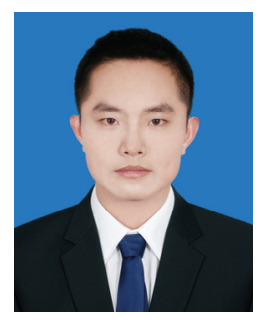

Bo Zhao He received Ph.D. degree in the Department of Control Science and Engineering from Jilin University, China in 2014. Now, he is a post-doctoral at Institute of Automation, Chinese Academy of Science, China. His interest covers fault diagnosis and fault tolerant control, and intelligent control.

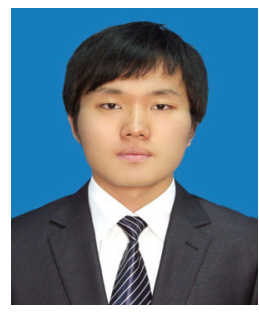

Chenghao Li He received M.E. degree in the Department of Control Science and Engineering from Jilin University, China in 2013. Now, he is a production engineer of FAW Car Co., Ltd. China. His interest covers fault diagnosis and fault tolerant control.

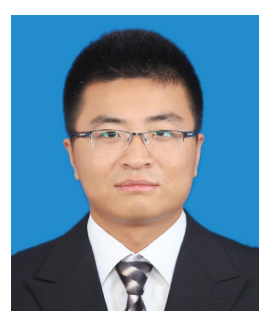

Tianhao Ma He received his M.E. degree in the Department of Control Science and Engineering from Changchun University of Technology, China in 2015. His interest covers intelligent control and robust control.

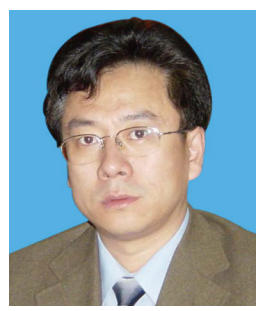

Yuanchun Li $\mathrm{He}$ received $\mathrm{Ph} . \mathrm{D}$. degree in the Department of General Mechanics from Harbin Institute of Technology, China in 1990. Now, he is a professor in the Department of Control Science and Engineering, Changchun University of Technology. His research interest covers complex system modeling and robot control. 